\title{
GENETIC EVALUATION AND CHARACTERIZATION OF JUTE (Corchorus spp. L) GENOTYPES USING DUS PARAMETERS
}

\author{
A. Das ${ }^{1}$ and D. Kumar \\ Central Research Institute for Jute \& Allied Fibres, Baarrackpore, India
}

\begin{abstract}
In this study 32 jute (Corchorus spp.) varieties comprised of 18 olitorius and 14 capsularis were evaluated and characterized for five quantitative and 12 qualitative characters as per revised official DUS Test Guidelines of Jute. The experimental materials were grown in a RBD with three replications at Baarrackpore, India during 2009-10. Significant differences among genotypes noted almost for almost all the quantitative traits. PCV and GCV were highest for 1000 seed weight (33.78 and $33.29 \%)$ followed by fibre fineness (27.11 and $26.70 \%$ ). Low variability was recorded in case of plant height. All the five characters exhibited more or less high heritability coupled with high genetic advance as per cent over mean. Out of 12 qualitative characters studied, in C. capsularis 8 traits were monomorphic, 4 traits were dimorphic. However, in C. olitorius no trait was monomorphic, 6 traits were dimorphic and 6 traits were polymorphic among varieties indicating their potential for varietal characterization.
\end{abstract}

Key words: Characterization, distinctiveness, heritability, jute, variability

\section{INTRODUCTION}

Cultivated species of jute, (Corchorus olitorius L. and C.capsularis L.) are distinct in their growth, branching habit and characteristics relating to leaf, flower, fruit, seed, bastfiber and photosensitivity (Ghosh, 1983). Genetic evaluation and characterization in both the cultivated species of jute have been reported by several workers but they studied the two area separately (Kumar et al., 2005 and Nayak and Baisakh, 2009). Success of plant breeding depends upon the nature and magnitude of variability present in the germplasm. Furthermore, the assessment of heritable and

\footnotetext{
${ }^{11}$ Assistant Professor, Bihar Agricultural University, College of Horticulture, Noorsarai, Nalanda, Bihar, India.

Corresponding author email: arpita_27das@rediffmail.com;
} 
non-heritable components of total variability will have immense value in the choice of suitable breeding procedure.

The "Protection of Plant Varieties and Farmers' Rights Act, 2001" (PPV\&FR Act, 2001) of India provides the opportunities for registration of new/extant varieties of agricultural crops including jute (Corchorus olitorius L. and C. capsularis L.) if it conforms to the criteria of DUS. Plant morphological characters have been recognized to constitute universally undisputed descriptors for DUS testing and varietal characterization of crop species. Use of morphological descriptors in sequential fashion is useful and convenient to distinguish different varieties. Qualitative characters being more stable over generations and environments are reliable for characterization of germplasm. Characterization of genotypes is useful to identify suitable lines and also to avoid duplication. Characterization of genotypes is also required for their protection under Plant Variety Protection (PVP) legislation, because varietal testing for Distinctiveness, Uniformity and Stability (DUS) is the basis for grant of protection of new plant varieties under the Protection of Plant Varieties and Farmers' Rights Act, 2001.

Since jute has been domesticated only around 200 years ago and many mutants have not yet been accumulated in jute population due to lack of human selection pressure for longer time (Mukherjee and Kumar, 2002), qualitative morphological characters of jute are mostly monomorphic and few are dimorphic and polymorphic. In India, while certain diagnostic features for released or notified jute varieties are known and used in seed certification (Kumar et al., 2005) the descriptors by and large are incomplete. The jute varieties have not so far been extensively described for various heritable morphological traits to enable the identification of these varieties and for unambiguous ascertainment of distinctness.

Hence, the present study was planned to genetically evaluate 32 varieties of jute for quantitative characters as per revised official DUS Test Guidelines of Jute (PPV\&FR Authority, 2008). Characterizations of genotypes of both the species were also carried out on the basis of qualitative morphological characters.

\section{MATERIALS AND METHODS}

The material for the present study comprised of 18 C.olitorius and 14 C.capsularis jute varieties. The experimental materials were grown in a randomized block design with three replications at the Central Research Institute for Jute \& Allied Fibres (22 $45^{\prime} \mathrm{N}$ and $88^{\circ} 26^{\prime} \mathrm{E}$ ), Baarrackpore, India during 2009-10 in plots of size $6.0 \times 1.60 \mathrm{~m}^{2}$ with 4 lines in each plot. Row to row and plant to plant distances were $40 \mathrm{~cm}$ and $7 \mathrm{~cm}$, respectively. Standard package of practices were followed to raise the crop (Kumar et al., 2008). Net plots were harvested at 120 days after sowing. The revised official DUS Test Guidelines of Jute (PPV\&FR Authority, 2008) suggested 17 simply observable and stable morphological characters to distinguish jute varieties. Among these five characters were quantitative and 12 characters were 
qualitative in nature. Data were recorded for five quantitative characters viz. plant height, time of $50 \%$ flowering, fibre strength, fibre fineness and 1000 seed weight. Plant height was recorded as height of the main stem measured from ground level to the point of forking at pre bud stage (before development of first flower). The character 'time of 50\% flowering' was noted when $50 \%$ of the plants had at least one open flower. Two fibre quality characters (fibre fineness and fibre strength) were observed after harvesting, retting and drying of fibre. Fibre fineness was measured by Airflow Fibre Fineness Tester (NIRJAFT, Kolkata) from three replicate samples by air flow method (Singh and Bandyopadhyay, 1968). Average fibre strength was determined by fibre bundle strength tester (NIRJAFT, Kolkata).

The genotypes were characterized for 12 distinguishing qualitative characters viz. premature flowering resistance, leaf lamina colour, leaf vein colour, leaf petiole colour, stipule colour, stem colour, leaf shape, pigmentation of calyx, basal stem root primordia, pod pigmentation, pod dehiscence and seed colour. The detail procedure for observation of these characters have been discussed in the revised official DUS Test Guidelines of Jute (PPV\&FR Authority, 2008). Observations were recorded on 10 randomly selected plants in each of three replications at specified stages of crop growth period when the characters under study had full expression. Genetic analysis was carried out using Genetic Model of INDOSTAT software (Indostat Statistical Software Package developed by Indostat Pvt. Ltd., Hyderabad, India).

\section{RESULTS AND DISCUSSION}

Analyses of variance revealed highly significant differences amongst genotypes for almost all the traits except fibre fineness and 1000 seed weight (Table 1). From the mean performance (Table 2) it was observed that highest plant height $(444.33 \mathrm{~cm})$ was observed in case of olitorius (var. JRO 66) followed var. JRO 878 $(443.33 \mathrm{~cm})$. Plant height of C. capsularis varieties is generally shorter than that of olitorius. Time of $50 \%$ flowering envisages the resistance to premature flowering. If a jute variety sown before middle of April does not flower prematurely in seedling stage and flowers at least after 100 days, the variety is known as premature flowering resistant variety (Ghosh, 1983). All the C. olitorius varieties except Chinsurah Green (97 days) were premature flowering resistant. Contrary to it, in C. capsularis all the varieties were resistant to premature flowering. In jute, fibre quality is judged by two parameters viz. fibre strength and fibre fineness. Among $C$. olitorius varieties fibre of JRO $36 \mathrm{E}$ (30.11 $\left.\mathrm{g} \mathrm{tex}^{-1}\right)$ followed by Chinsurah Green (27.82 $\left.\mathrm{g} \mathrm{tex}^{-1}\right)$ were strongest and fibre of Bidhan Rupali exhibited lowest value (16.08 $\left.\mathrm{g} \mathrm{tex}^{-1}\right)$. Among $C$. capsularis varieties, JRC 698 had strongest fibre $\left(25.73 \mathrm{~g} \mathrm{tex}^{-1}\right)$. In case of fibre fineness lower is the value means finer is the fibre. C. capsularis variety JRC 80 (1.38 tex) followed by Bidhan Pat 1 (1.53 tex) were the finest variety and in case of C. olitorius variety, JRO 620 (2.57 tex) followed by JRO 128 (2.59 tex) possessed the finest position. Regarding 1000 seed weight $C$. capsularis varieties are generally larger than C. olitorius ones. 
From the table 3 it was found that phenotypic variance was higher than genotypic variance in all the characters. But the difference between PCV and GCV was very close which resemble the finding of Islam et al., 2002. The PCV and GCV was found to be highest for 1000 seed weight (g) (33.78 \% and $33.29 \%$ ) followed by fibre fineness (tex) (27.11 \% and $26.70 \%)$. This indicated presence of more variability which gives scope for improvement of these traits by selection. Low variability was recorded in case of plant height $(14.58 \%$ and $14.16 \%)$ thereby emphasizing the need for generating more variability for this character. The variety having different plant height group could be effectively utilized in developing genotypes with better plant height because in case of jute it is the most important yield contributing character. GCV values only are not enough to determine the genetic variability, this could be done with the help of heritability and genetic advance estimates to assess the heritable portion of total variation and extent of expected portion of genetic gain under selection. High heritability was recorded for time of 50\% flowering (99\%) followed by fibre fineness and seed weight (97\%). This result corroborated with the finding of Ghosh Dastidar et al., 1993. Time of 50\% flowering exhibited low genetic advance (46.83) as compared with 1000 seed weight (86.61) and fibre fineness (69.40). Similar results have been reported by Islam et al., 2002.High heritability coupled with high genetic advance in almost all the characters indicated the presence of additive gene effect, so their improvement can be done through mass selection.

The characterization of genotypes (Table 4) revealed wide variation for all the qualitative characters among the genotypes. Earlier reports by Kumar et al., 2005 have also shown the presence of variation for these qualitative traits in jute genotypes. Out of 12 morphological characteristics studied, in C. capsularis 8 traits (premature flowering resistance, leaf lamina colour, leaf vein colour, stipule colour, leaf shape, basal stem root primordia, pod dehiscence and seed colour) were found to be monomorphic, 4 traits (leaf petiole colour, stem colour, pigmentation of calyx and pod pigmentation) were dimorphic. In $C$. olitorius no trait was monomorphic, 6 traits (premature flowering resistance, leaf lamina colour, leaf shape, pigmentation of calyx, basal stem root primordial and pod dehiscence) were dimorphic and 6 traits (leaf vein colour, leaf petiole colour, stipule colour, stem colour, pod pigmentation and seed colour) were polymorphic among varieties indicating their potential for varietal characterization.Similar attempts for characterization as per DUS guidelines were made in oat (Kumar et al., 2002), pearl millet (Kumar et al., 2004), rice (Joshi et al., 2007), jute (Kumar and Begum, 2008) and maize (Yadav and Singh, 2010).

The study revealed sufficient genetic variability both for qualitative and quantitative morphological characters among the varieties, which can be exploited for varietal improvement. Therefore, a gene pool can be generated by crossing the variety of interest which could be further used as a source material to develop promising varieties. The characterization of genotypes as per DUS descriptors could be used effectively for identification and grouping of the jute varieties and varieties 
satisfying the DUS criteria for these descriptors could be registered under the PPV\&FR Act for obtaining Plant Breeders and Farmers' Rights.

\section{REFERENCES}

Ghosh, Dastidar., K.K., Agarwalla, K.K. and Roychowdhury, P. 1993. Genetic variability and association of component characters for seed yield in C. olitorus jute. Indian J Genet., 53: $157-160$.

Ghosh, T. 1983. Handbook on Jute, FAO Plant Production and Protection, paper no. 51, Rome, Italy, pp. 29-31.

Islam, M.R., Islam, M.M., Akter, N. and Ghosh, R.K. 2002. Genetic variability and performance of tossa jute. Pakistan J. Biol. Sci., 5: 744-745.

Joshi, M.A., Sarao, N.K., Sharma, R.C., Singh, P. and Bharaj, T.S. 2007. Varietal characterization of rice (Oryza sativa L.) bases morphological descriptors. Seed Res., 35: 188-193.

Kumar, D., Agrawal, R.C. and Begum, T. 2008. Analysis for identification of distinct and uniform extant jute (Corchorus olitorius L. and C. capsularis L.) varieties. Seed Res., 36: 121-134.

Kumar, M.B.A., Sherry, R.J., Dadlani, M., Varier, A. and Sharma, S.P. 2004. Characterization of pearl millet hybrids and parental lines using morphological characters. Seed Res., 32: 15-19.

Kumar, Y., Ram, H., Gulia, S.K. 2002. Varietal identification in oat based on field parameters. Forage Res., 28: 73-76.

Kumar, D., Das, A., Mahato, P., Lakshman, S.S. and Mandi, S. 2005. Identification key for notified varieties and varieties of common knowledge of jute (Corchorus olitorius L. and C. capsularis L.). Indian J. Genet., 65: 236-238.

Mukherjee, S. and Kumar, D. 2002. Screening of tossa jute (Corchorus olitorius L.) germplasm for male sterility. Indian J Genet., 62: 361-362.

Nayak, B. K. and Baisakh, B. 2009. Genetic analysis for fiber yield and its components in tossa jute (Corchorus olitorius L.). Envir and Ecol., 27: 86-88

PPV \& FR ACT, 2001. Protection of Plant Varieties and Farmers' Rights Act (No. 53 of 2001). Dept. of Agriculture and Cooperation, Ministry of Agriculture, Govt. of India, Krishi Bhavan, New Delhi.

PPV \& FR Authority, 2008. Guidelines for the conduct of test for Distinctiveness, Uniformity and Stability on jute (Corchorus olitorius L. and C. capsularis L.). Plant Variety J. India., 2: 1-29.

Singh, N.G. and Bandyopadhyay, S.P. 1968. An airflow method for the determination of the fibre fineness of jute and mesta. J. Textile Instt., 59: 148-156.

Yadav, V.K. and Singh, I.S. 2010. Comparative evaluation of maize inbred lines (Zea mays L.) according to DUS testing using morphological, physiological and molecular markers. Agric Sci., 1: 131-142 
Table 1: Analysis of variance for five quantitative characters in 32 varieties of jute

\begin{tabular}{|c|c|c|c|c|c|c|}
\hline \multirow{2}{*}{$\begin{array}{l}\text { Sources of } \\
\text { Variation }\end{array}$} & \multirow[t]{2}{*}{ d.f. } & \multicolumn{5}{|c|}{ Mean Squares } \\
\hline & & $\begin{array}{c}\text { Plant height } \\
\text { (cm) }\end{array}$ & $\begin{array}{c}\text { Time of } 50 \\
\text { \% flowering } \\
\text { (days) }\end{array}$ & $\begin{array}{c}\text { Fibre } \\
\text { strength } \\
\left(\text { g tex }^{-1}\right)\end{array}$ & $\begin{array}{l}\text { Fibre fineness } \\
\text { (tex) }\end{array}$ & $\begin{array}{l}1000 \text { Seed } \\
\text { weight (g) }\end{array}$ \\
\hline Replication & 2 & $141.27 * *$ & 5.01 & 5.01 & 0.02 & 0.01 \\
\hline Treatment & 31 & $7650.99 * *$ & $1474.47 * *$ & $46.95 * *$ & 1.17 & 2.19 \\
\hline Error & 62 & $151.02 * *$ & 2.33 & 0.57 & 0.01 & 0.02 \\
\hline
\end{tabular}

** Significant at $1 \%$ level

Table 2: Mean performance and variability components of 32 jute varieties for five quantitative characters

\begin{tabular}{|c|c|c|c|c|c|}
\hline Varieties & Plant height (cm) & $\begin{array}{c}\text { Time of } 50 \% \\
\text { flowering (days) }\end{array}$ & $\begin{array}{l}\text { Fibre strength } \\
\quad\left(\mathrm{g} \mathrm{tex}^{-1}\right)\end{array}$ & $\begin{array}{l}\text { Fibre fineness } \\
\text { (tex) }\end{array}$ & 1000 Seed weight (g) \\
\hline \multicolumn{6}{|c|}{ C. olitorius varieties } \\
\hline JRO 632 & 405.33 & 108.33 & 25.70 & 2.99 & 2.17 \\
\hline JRO 3690 & 413.66 & 122.00 & 20.55 & 2.67 & 1.77 \\
\hline KOM 62 & 313.33 & 130.00 & 20.09 & 2.82 & 1.83 \\
\hline TJ 40 & 430.66 & 129.33 & 18.25 & 3.09 & 1.70 \\
\hline JRO 66 & 444.33 & 134.67 & 27.13 & 3.03 & 1.83 \\
\hline JRO 524 & 397.00 & 157.00 & 26.87 & 3.37 & 1.83 \\
\hline JRO 7835 & 411.66 & 157.67 & 26.85 & 3.45 & 1.77 \\
\hline JRO 878 & 443.33 & 141.00 & 26.18 & 2.62 & 1.70 \\
\hline JRO 8432 & 364.00 & 148.67 & 27.55 & 2.82 & 2.13 \\
\hline JRO 128 & 364.00 & 158.00 & 27.76 & 2.59 & 1.80 \\
\hline S-19 & 402.66 & 144.33 & 26.96 & 2.63 & 1.50 \\
\hline BidhanRupali & 392.00 & 109.67 & 16.08 & 2.70 & 1.93 \\
\hline JRO 620 & 410.00 & 129.67 & 26.55 & 2.57 & 1.73 \\
\hline Chinsurah Green & 314.00 & 97.45 & 18.82 & 2.86 & 2.17 \\
\hline Sudan Green & 306.00 & 109.00 & 25.08 & 2.96 & 2.17 \\
\hline Tanganyika 1 & 301.00 & 108.33 & 24.93 & 2.92 & 1.77 \\
\hline JRO 36E & 406.00 & 156.00 & 30.11 & 2.99 & 1.77 \\
\hline JRO 2345 & 376.67 & 140.67 & 24.63 & 2.59 & 1.70 \\
\hline \multicolumn{6}{|c|}{ C. capsularis varieties } \\
\hline JRC 212 & 326.66 & 93.67 & 23.62 & 1.84 & 3.37 \\
\hline JRC 7447 & 335.00 & 114.00 & 23.50 & 1.90 & 3.20 \\
\hline JRC 321 & 303.00 & 88.33 & 22.52 & 1.53 & 3.90 \\
\hline Padma & 321.33 & 104.67 & 24.50 & 2.53 & 3.43 \\
\hline JRC 4444 & 356.67 & 127.67 & 22.37 & 1.97 & 3.13 \\
\hline UPC 94 & 323.67 & 98.00 & 22.92 & 1.70 & 3.83 \\
\hline JRC 698 & 342.00 & 114.67 & 25.74 & 1.83 & 3.17 \\
\hline Bidhan Pat 1 & 340.00 & 101.00 & 18.47 & 1.53 & 3.30 \\
\hline Bidhan Pat 2 & 326.00 & 94.00 & 20.30 & 2.03 & 3.26 \\
\hline Bidhan Pat 3 & 334.67 & 109.67 & 19.69 & 1.77 & 3.47 \\
\hline JRC 80 & 285.67 & 163.67 & 20.14 & 1.38 & 3.77 \\
\hline KC 1 & 277.33 & 147.33 & 22.06 & 2.57 & 4.17 \\
\hline KTC 1 & 275.33 & 136.00 & 22.66 & 2.43 & 3.33 \\
\hline D 154 & 283.00 & 109.67 & 22.54 & 2.50 & 3.30 \\
\hline
\end{tabular}


Table 3. Mean, range and components of variability for five quantitative traits in 32 genotypes of jute

\begin{tabular}{|l|c|c|c|c|c|c|}
\hline \multicolumn{1}{c|}{ Character } & Mean \pm SE & Range & PCV (\%) & $\begin{array}{c}\text { GCV } \\
\mathbf{( \% )}\end{array}$ & $\begin{array}{c}\mathbf{h} \\
\mathbf{( \% )}\end{array}$ & $\begin{array}{c}\text { GA as \% } \\
\text { over } \\
\text { Mean }\end{array}$ \\
\hline Plant height (cm) & $353.23 \pm 6.98$ & $444.33-275.33$ & 14.58 & 14.16 & 94 & 36.29 \\
$\begin{array}{l}\text { Days to flowering } \\
\text { (days) }\end{array}$ & $124.59 \pm 0.87$ & $163.67-93.67$ & 17.82 & 17.78 & 99 & 46.83 \\
Fibre strength (g/tex) & $21.74 \pm 0.43$ & $27.76-15.66$ & 18.42 & 18.08 & 96 & 46.89 \\
Fibre fineness(tex) & $2.33 \pm 0.06$ & $1.38-3.45$ & 27.11 & 26.70 & 97 & 69.40 \\
Seed weight (g) & $2.56 \pm 0.08$ & $4.16-1.50$ & 33.78 & 33.29 & 97 & 86.61 \\
\hline
\end{tabular}

Table 4. Distinguishing qualitative characters of the genotypes as per Jute DUS Test Guidelines

\begin{tabular}{|c|c|c|c|c|c|c|c|c|c|c|c|c|}
\hline Variety & PFR & LLC & LVC & LPC & SC & StC & LS & PC & BSRP & PP & PD & SdC \\
\hline \multicolumn{13}{|c|}{ C. olitorius varieties: } \\
\hline JRO-632 & Absent & Green & Green & Green & Green & Green & Ovatelanceolate & Green & Absent & Green & Present & Steel grey \\
\hline JRO-3690 & Absent & Green & Green & Green & Green & Green & Ovatelanceolate & Green & Absent & Green & Present & Steel grey \\
\hline КОМ-62 & Absent & Green & Green & Red & Red & Purple & Ovatelanceolate & Green & Absent & Red & Present & Steel grey \\
\hline TJ-40 & Absent & Green & Green & Green & Green & Green & Ovatelanceolate & Green & Absent & Green & Present & Green \\
\hline JRO-66 & Absent & Green & Green & Green & Green & Green & Ovatelanceolate & Green & Absent & Green & Absent & Steel grey \\
\hline JRO-524 & Present & Green & Green & Green & Green & Green & Ovatelanceolate & Green & Absent & Green & Absent & Black \\
\hline JRO-7835 & Present & Green & Green & Green & Green & Green & Ovatelanceolate & Green & Present & Green & Absent & Black \\
\hline JRO-878 & Present & Green & Red & Red & Red & Purple & Ovatelanceolate & Green & Absent & Red & Absent & Black \\
\hline JRO-8432 & Present & Green & Green & Green & Green & Green & Ovatelanceolate & Green & Absent & Green & Absent & Black \\
\hline $\begin{array}{l}\text { JRO-128 } \\
\text { S-19 }\end{array}$ & $\begin{array}{l}\text { Present } \\
\text { Present }\end{array}$ & $\begin{array}{l}\text { Green } \\
\text { Green }\end{array}$ & $\begin{array}{l}\text { Green } \\
\text { Red }\end{array}$ & $\begin{array}{l}\text { Green } \\
\text { Red }\end{array}$ & $\begin{array}{l}\text { Green } \\
\text { Red }\end{array}$ & $\begin{array}{l}\text { Green } \\
\text { Red }\end{array}$ & $\begin{array}{l}\text { Ovatelanceolate } \\
\text { Ovatelanceolate }\end{array}$ & $\begin{array}{l}\text { Green } \\
\text { Green }\end{array}$ & $\begin{array}{l}\text { Absent } \\
\text { Absent }\end{array}$ & $\begin{array}{l}\text { Green } \\
\text { Red }\end{array}$ & $\begin{array}{l}\text { Absent } \\
\text { Absent }\end{array}$ & $\begin{array}{c}\text { Black } \\
\text { Steel grey }\end{array}$ \\
\hline $\begin{array}{l}\text { BidhanRupali } \\
\text { JRO-620 }\end{array}$ & $\begin{array}{l}\text { Absent } \\
\text { Absent }\end{array}$ & $\begin{array}{l}\text { Pale Green } \\
\text { Green }\end{array}$ & $\begin{array}{l}\text { Pale Green } \\
\text { Red }\end{array}$ & $\begin{array}{l}\text { Pale Green } \\
\text { Red }\end{array}$ & $\begin{array}{l}\text { Pale Green } \\
\text { Red }\end{array}$ & $\begin{array}{l}\text { Pale Green } \\
\text { Purple }\end{array}$ & $\begin{array}{l}\text { Ovatelanceolate } \\
\text { Lanceolate }\end{array}$ & $\begin{array}{l}\text { Pale Green } \\
\text { Green }\end{array}$ & $\begin{array}{l}\text { Absent } \\
\text { Absent }\end{array}$ & $\begin{array}{l}\text { Pale Green } \\
\text { Red }\end{array}$ & $\begin{array}{l}\text { Present } \\
\text { Present }\end{array}$ & $\begin{array}{l}\text { Steel grey } \\
\text { Steel grey }\end{array}$ \\
\hline Chinsurah Green & Absent & Green & Green & Green & Green & Green & Ovatelanceolate & Green & Absent & Green & Present & Green \\
\hline Sudan Green & Present & Green & Green & Green & Green & Green & Ovatelanceolate & Green & Absent & Green & Absent & Green \\
\hline Tanganyika-1 & Present & Green & Green & Green & Green & Green & Ovatelanceolate & Green & Absent & Green & Absent & Green \\
\hline \begin{tabular}{|l} 
JRO-36E \\
JRO-2345 \\
\end{tabular} & $\begin{array}{l}\text { Present } \\
\text { Present }\end{array}$ & $\begin{array}{l}\text { Green } \\
\text { Green } \\
\end{array}$ & $\begin{array}{l}\text { Green } \\
\text { Green }\end{array}$ & $\begin{array}{l}\text { Green } \\
\text { Green } \\
\end{array}$ & $\begin{array}{l}\text { Green } \\
\text { Green }\end{array}$ & $\begin{array}{l}\text { Green } \\
\text { Green } \\
\end{array}$ & $\begin{array}{l}\text { Ovatelanceolate } \\
\text { Ovatelanceolate }\end{array}$ & $\begin{array}{l}\text { Green } \\
\text { Green } \\
\end{array}$ & $\begin{array}{l}\text { Absent } \\
\text { Absent }\end{array}$ & $\begin{array}{l}\text { Green } \\
\text { Green } \\
\end{array}$ & $\begin{array}{l}\text { Absent } \\
\text { Absent }\end{array}$ & $\begin{array}{c}\text { Steel grey } \\
\text { Green }\end{array}$ \\
\hline \multicolumn{13}{|c|}{ C. capsularis varieties } \\
\hline JRC-212 & Present & Green & Green & Green & Green & Green & Ovatelanceolate & Green & Absent & Green & Absent & $\begin{array}{l}\text { Chocolate } \\
\text { brown } \\
\text { Cocolate }\end{array}$ \\
\hline JRC-7447 & Present & Green & Green & Green & Green & $\begin{array}{l}\text { Green } \\
\text { Coppery }\end{array}$ & Ovatelanceolate & Green & Absent & Green & Absent & $\begin{array}{c}\text { brown } \\
\text { Chocolate }\end{array}$ \\
\hline JRC-321 & Present & Green & Green & Red & Green & $\begin{array}{l}\text { red } \\
\text { Coppery }\end{array}$ & Ovatelanceolate & Red & Absent & Red & Absent & $\begin{array}{c}\text { brown } \\
\text { Chocolate }\end{array}$ \\
\hline Padma & Present & Green & Green & Red & Green & red & Ovatelanceolate & Red & Absent & Red & Absent & $\begin{array}{c}\text { brown } \\
\text { Chocolate }\end{array}$ \\
\hline JRC-4444 & Present & Green & Green & Green & Green & $\begin{array}{c}\text { Green } \\
\text { Coppery }\end{array}$ & Ovatelanceolate & Green & Absent & Green & Absent & $\begin{array}{c}\text { brown } \\
\text { Chocolate }\end{array}$ \\
\hline UPC-94 & Present & Green & Green & Red & Green & red & Ovatelanceolate & Red & Absent & Red & Absent & $\begin{array}{c}\text { brown } \\
\text { Chocolate }\end{array}$ \\
\hline JRC-698 & Present & Green & Green & Green & Green & Green & Ovatelanceolate & Green & Absent & Green & Absent & $\begin{array}{c}\text { brown } \\
\text { Chocolate }\end{array}$ \\
\hline Bidhan Pat-1 & Present & Green & Green & Green & Green & Green & Ovatelanceolate & Green & Absent & Green & Absent & $\begin{array}{c}\text { brown } \\
\text { Chocolate }\end{array}$ \\
\hline Bidhan Pat-2 & Present & Green & Green & Green & Green & Green & Ovatelanceolate & Green & Absent & Green & Absent & $\begin{array}{c}\text { brown } \\
\text { Chocolate }\end{array}$ \\
\hline Bidhan Pat-3 & Present & Green & Green & Green & Green & Green & Ovatelanceolate & Green & Absent & Green & Absent & $\begin{array}{c}\text { brown } \\
\text { Chocolate }\end{array}$ \\
\hline JRC-80 & Present & Green & Green & Green & Green & Green & Ovatelanceolate & Green & Absent & Green & Absent & $\begin{array}{c}\text { brown } \\
\text { Chocolate }\end{array}$ \\
\hline KC-1 & Present & Green & Green & Green & Green & Green & Ovatelanceolate & Green & Absent & Green & Absent & $\begin{array}{c}\text { brown } \\
\text { Chocolate }\end{array}$ \\
\hline KTC-1 & Present & Green & Green & Green & Green & Green & Ovatelanceolate & Green & Absent & Green & Absent & $\begin{array}{c}\text { brown } \\
\text { Chocolate }\end{array}$ \\
\hline D-154 & Present & Green & Green & Red & Green & Green & Ovatelanceolate & Red & Absent & Red & Absent & brown \\
\hline
\end{tabular}

PFR: premature flowering resistance. LLC: leaf lamina colour. LVC: leaf vein colour. LPC: leaf petiole colour. SC: stipule colour. StC: stem colour. LS: leaf shape.

PC: pigmentation of calyx, BSRP: basal stem root primordia. PP: pod pigmentation. PD: pod dehiscence. SdC: seed colour. 